\title{
Using non-organic resist based on As-S-Se chalcogenide glasses for combined optical/digital security devices
}

\author{
S.A. Kostyukevych ${ }^{a}$, N.L. Moskalenko ${ }^{a}$, P.E. Shepeliavyi ${ }^{a}$, V.I. Girnyk ${ }^{b}$, \\ I.V. Tverdokhleb ${ }^{c}$ A.A. Ivanovsky ${ }^{c}$ \\ anstitute of Semiconductor Physics, 41 prosp. Nauki, 03028 Kiev, Ukraine \\ ${ }^{b}$ Optronics Ltd., 03127 Kiev, PB 610, Ukraine \\ ${ }^{c}$ Radiophysical Dept., Kiev T. Shevchenko Natiomnal University, 2 prosp. Akademika Glushkova, 03187 Kiev, Ukraine
}

\begin{abstract}
Modern holographic protective elements used as emblems against counterfeiting are being more complicated as they should oppress criminal world. 2D, 3D, 3D rainbow holograms or simple diffraction structures protecting documents can not be acceptable against illegal copying of important documents, bank notes or valuable products. Recent developments in technology of Optical variable devices permit world leaders to create more advanced security elements: Kinegrams ${ }^{\mathrm{TM}}$, Exelgrams ${ }^{\mathrm{TM}}$, Pixelgrams ${ }^{\mathrm{TM}}$, Kineforms ${ }^{\mathrm{TM}}$. These products are used for protecting the most confidential documents and bank notes, but now even their security level can not be enough, and besides their automatic identification is vulnerable to factors of instability. We elaborate new visual security devices based on the usage of expensive and advanced technology of Combined optical/digital security devices. The technology unites digital and analog methods of synthesis and recording Visual security devices. The analog methods include technique of optical holography - different combinations of $2 \mathrm{D} / 3 \mathrm{D}, 3 \mathrm{D}, 2 \mathrm{D} /$ $3 \mathrm{D}+3 \mathrm{D}$ structures. Basing on them the design with elements of 3D graphic including security elements and hidden machine-readable images are implemented. The digital methods provide synthesis of Optical Variable Devices including special security elements, computer generated holograms and Kineforms ${ }^{\mathrm{TM}}$. Using them we create determined and quasi-random machinereadable images. Recording are carried out using the combined optical and electron-beam submicrometer technology. The results obtained show effectiveness of the combined technology permitting to increase the security level essentially, which should increase tamper and counterfeit resistance for many years.
\end{abstract}

Keywords: visual security devices, combined security devices, optical diffraction devices, computer generated holograms, kineform, automatic identification systems, documents.

Paper received 29.01.01; revised manuscript received 14.02.01; accepted for publication 16.02.01.

\section{Introduction}

Modern holographic security devices used as emblems against counterfeiting are being more difficult as they should oppress criminal world. 2D/3D, 3D rainbow holograms or simple diffraction structures protecting documents can not be acceptable against illegal copying. Now these simple Visual security devices (VSDs) are manufactured easily in hundreds of holographic companies worldwide and are not considered as sufficient security for important documents, bank notes or valuable products. Further technology development of VSDs permits leaders among holographic companies to offer more difficult VSDs. For example, high resolution emblems including diffraction gratings using spatial and frequency encoding. But today, when opened market offers dot-matrix systems with high resolution (Digital Matrix Corp., USA; Westmead Technology Ltd., UK), they become commercially available for several holographic companies and are not unique. Another aspect of this problem is automatic identification of VSDs. The mostly cases for VSDs authenticity examining uses visual analysis forced in need by optical devices (microscope, etc.). Despite it is not perfect it will be used widely by protection of bank notes, travel documents, commercial goods, etc. In all cases when automatic identification can not be implemented by economic of technical conditions. In our opinion the synthesis of easily recognition, dynamic images created by difficult super technologies can solve this problem.

The recent achievements in VSDs technology - Optical variable devices (OVDs) permits to world leaders to create more perfect technologies: Kinegrams ${ }^{\mathrm{TM}}$ (Landis 


\section{S.A. Kostyukevych et al.: Using non-organic resist based on As-S-Se chalcogenide ...}

\& Gyr ${ }^{1,2}$ Corp., Switzerland), Exelgrams ${ }^{\text {TM }}$, Pixelgrams $^{\text {TM }}$ (CSIRO $^{3,4}$, Australia), Kineforms (Light Impressions Europe, UK). Their products are used for security of the mostly valuable documents and bank notes. All these four OVD types are created by using of interactive gratings, their images are created by computer, they contain kinematic effects reconstructed by scattered light in majority of view angles, their manufacturing is possible by the usage of super technologies. But even their security level is not satisfactory up to date and their automatic identification is weak to action of different destabilizing factors.

The last developments in OVD technologies presents a new direction - usage of structures calculated by numeric methods, both gratings and computer generated holograms. These methods permits synthesis of digital OVD that could contain machine readable information (Warsaw University of Technology, Warszawa, Poland; Holographia Polska; Wroclaw, Poland; Weizmann Institute of Science, Israel; Landis \& Gyr Communication Corporation, Zug, Switzerland).

Within the framework of our work we considered the method of synthesis and recording the Combined optical/digital security devices (CO/DSDs) elaborated by Optronics Ltd. These CO/DSDs unites organically two parts - optical and digital. The optical one consists of elements of optical holography that include different combinations of 2D, 3D, 2D/3D structures. It creates design with elements of 3D graphics including security elements as optically variable guilloche, optically variable microtexts, microlines, difficult background patterns based on random processes and fractal geometry, hidden machine readable images. The digital part consists of synthesis of OVDs including special protective elements: cinematic effects, microtext, submicrotext, computer generated holograms, kineform elements, determined and random machine readable elements.

\section{Synthesis and recording CO/DSD}

Manufacturing CO/DSD combigrams is a difficult technological process requiring particular attention to design of optical and digital parts of an emblem, their co-ordination and further recording. As registering media we investigated non-organic resist of $\mathrm{As}_{60} \mathrm{~S}_{60-\mathrm{x}} \mathrm{Se}_{\mathrm{x}}(x=20), 30,40$ type. Principally, two methods of $\mathrm{CO} / \mathrm{DSD}$ recording are available: 1) method of superimposed exposure (two-exposure method) and 2) spatially-splitted exposure method (multiplexed method). Both of these methods can be used for $\mathrm{CO} / \mathrm{DSD}$ practical synthesis and recording.

\subsection{Two-exposure method}

The two-exposure method is the most attractive from the viewpoint of its simplicity but requires thorough design accuracy as well as careful control of exposure and irradiation doses. The main recording regimes and their sequence by $\mathrm{CO} / \mathrm{DSD}$ synthesis are following:

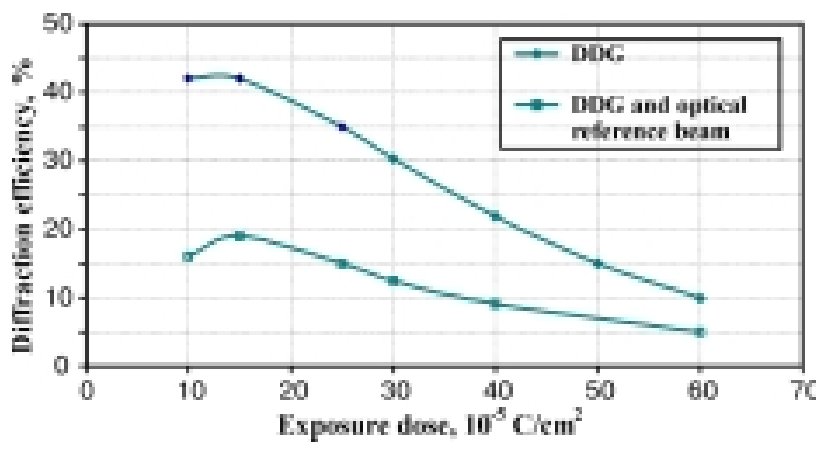

Fig. 1. Diffraction efficiency of DDG as a function of exposure doze by one-exposure recording of DDG (curve 1) and twoexposure recording of DDG and optical reference beam with exposure energy of $60 \mathrm{~mJ} / \mathrm{cm}^{2}$ (curve 2).

1) digital part (Digital Diffraction Grating - DDG) is recorded on the exposure field by a reference beam;

2) digital part of CO/DSD is recorded on optical hologram (Optical Diffraction Grating - ODG);

3) order of DDG and ODG recording can be reverse.

We investigated influence of optical reference beam on diffraction efficiency (DE) of the ODG (Fig. 1)

In linear approach, limited by determined dynamic range of relief-exposure characteristics of $\mathrm{As}_{60} \mathrm{~S}_{60-\mathrm{x}} \mathrm{Se}_{\mathrm{x}}$, this dependence does not exceed the classical theory bounds. But practically an essential role on curve 2 (Fig. 1) effects interpolation of points of digital hologram by its recording by electron beam lithography (EBL) photoresist system. Microrelief picture of CO/DSD part with superimposed exposure of DDG + reference beam (Fig. 2) testifies it.

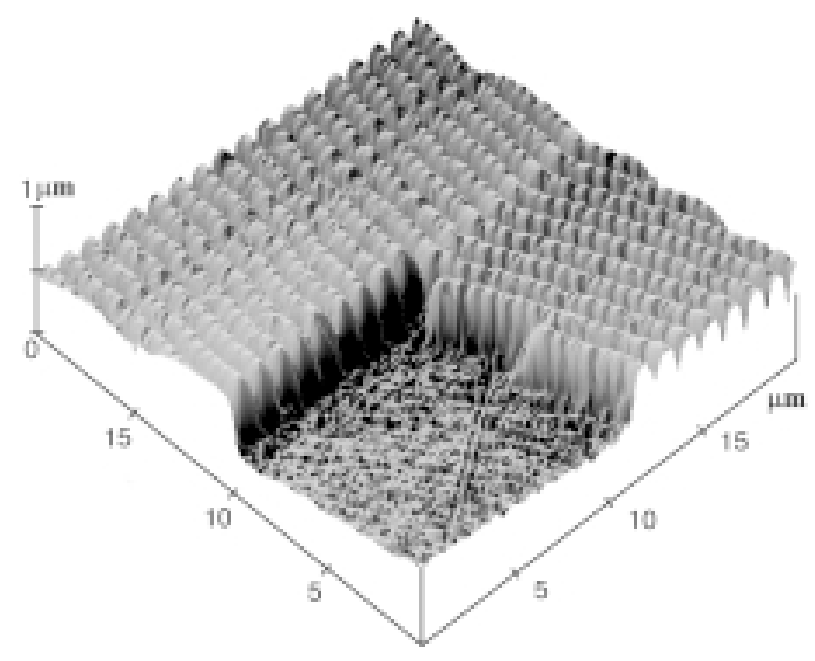

Fig. 2. Microstructure of CO/DSD surface: part of superimposed exposure of digital hologram + optical background. 


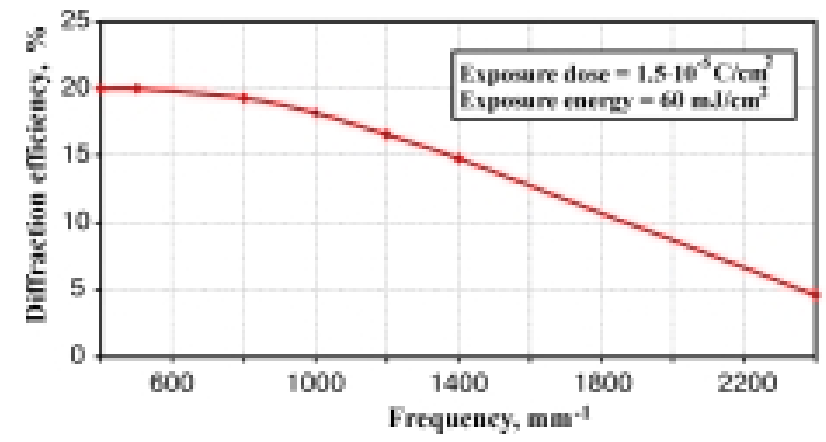

Fig. 3. Diffraction efficiency of DDG as a function of spatial frequency by two-exposures recording of DDG and optical reference beam.

Frequency-contrast curve of a system (Fig. 3) does not depend on superimposed exposure of reference beam and by necessity is corrected by software method.

The most interesting is a case of simultaneous recording optical and digital holograms, i. e. pure superimposed exposure. Curves on Fig. 4 shows dependence of optical and digital grating on order of their recording and exposure dose. We can see that choosing a necessary level of exposure dose of digital hologram one can obtain reconstruction of both holograms simultaneously or only digital one. Fig. 5 shows a mechanism of such recording.

By this digital and optical hologram are appeared on different levels of registering media with shift of $0.4 \div$ $\div 0.5 \mu \mathrm{m}$ (Fig. 6).

Recording regime choice is determined proceeding from a structure and design of a concrete CO/DSD. We considered three cases of digital and optical holograms interaction and can conclude that we achieved totally controlled process of recording of each of these parts per-

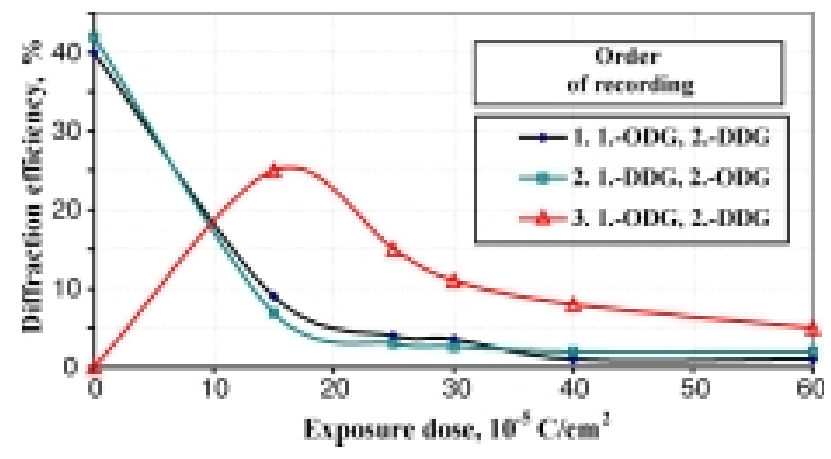

Fig. 4. Diffraction efficiency of $\operatorname{ODG}(1,2)$ with frequency of $900 \mathrm{~mm}^{-1}$ and DDG (3) with frequency of $909 \mathrm{~mm}^{-1}$ and exposure energy of $60 \mathrm{~mJ} / \mathrm{sm}^{2}$ as a function of exposure dose of DDG with frequency of $909 \mathrm{~mm}^{-1}$ by different order of their recording.

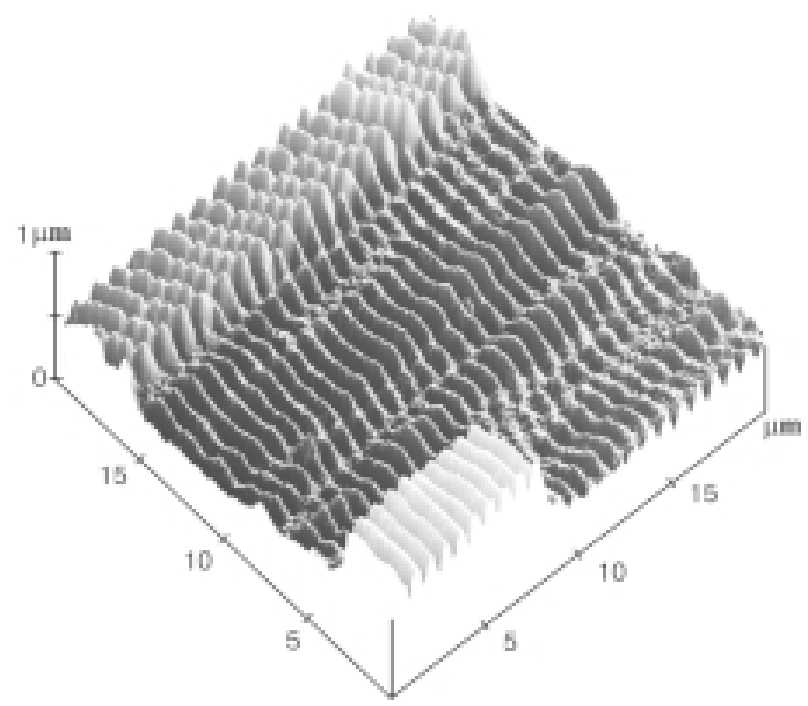

Fig. 5. Microstructure of CO/DSD surface: part of superimposed exposure of optical + digital hologram.

mitting obtaining of high diffraction efficiency for both optical and digital holograms.

\subsection{Multiplexed method of CO/DSD recording}

This method of spatially splitted exposure consisted of formation of optical and digital parts of CO'DSD on different places of registering medium. This is more simple case from the viewpoint of recording methods and spatial co-ordination of optical and digital parts of CO/DSD. We investigated influence of electron recording on optical one.

Fig. 7 shows that even by maximal exposure dose zone of influence does not exceed $4 \div 5 \mu \mathrm{m}$ (for setups with electron energy $20 \mathrm{kV}$ ). We measured optical hologram of CO/DSD on $>5 \mu \mathrm{m}$ distances. Fig. 8 allows to consider recording of digital and optical parts of CO/DSD independently.

\section{Security properties of CO/DSD}

CO/DSDs are based on combined technologies including maximal number of security degrees. One of ubmicrotext sample is shown in Fig. 8.

This is a three-level structure: optical hologram, digital hologram and submicrotext as a third level.

CO/DSDs are designed at first for valuable papers, government documents and personal identification document protection.

The base of effective anti-counterfeiting Optronics $\mathrm{CO} / \mathrm{DSD}$ system is a strategy of multi-level inspection. $\mathrm{CO} / \mathrm{DSD}$ structure consists of several security degrees on macro- and microlevels that are easily identificated in determined places including manufacturing, distribution and using of the products to be protected. 
S.A. Kostyukevych et al.: Using non-organic resist based on As-S-Se chalcogenide ...

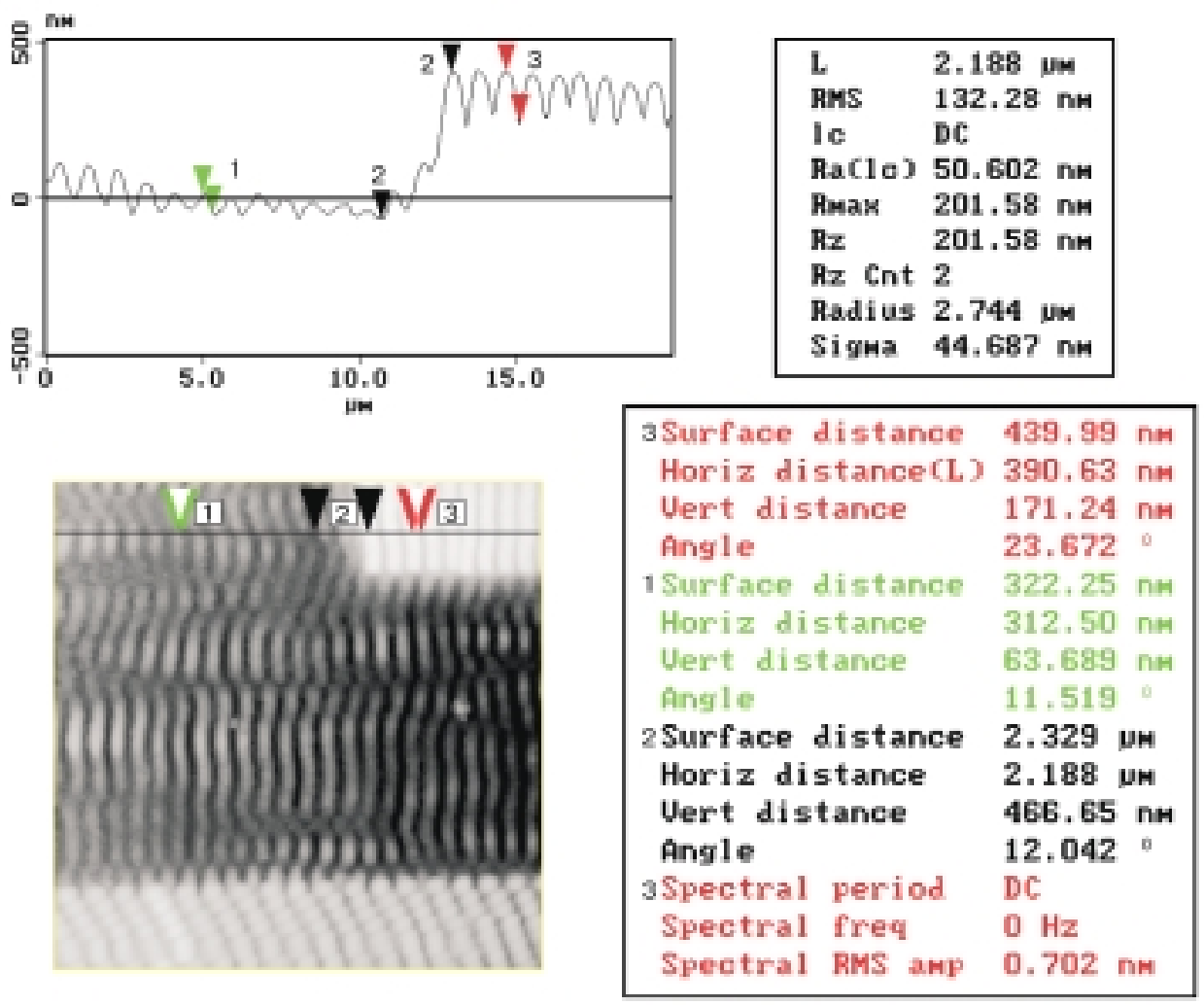

Fig. 6. Microstructure of CO/DSD surface: part of superimposed exposure of optical + digital hologram. Section analysis.

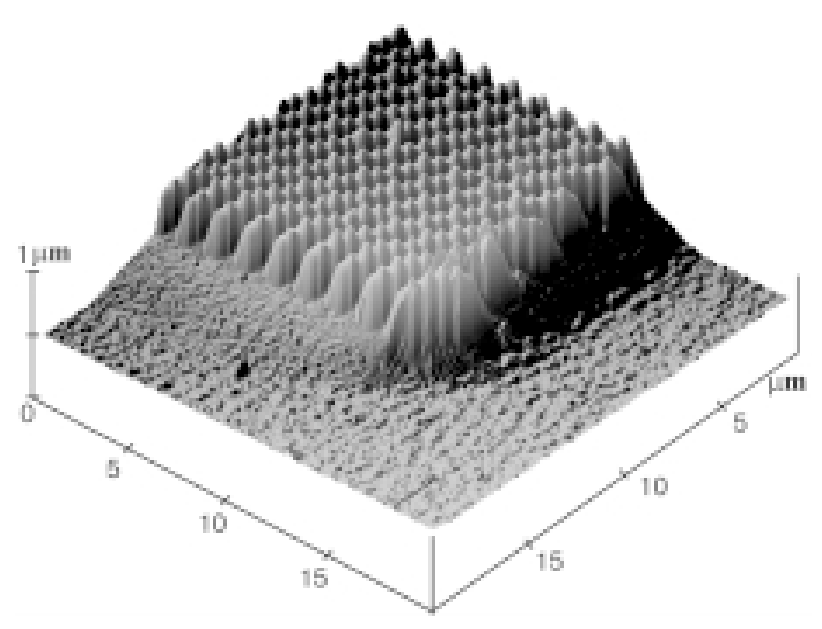

Fig. 7. Microstructure of CO/DSD surface: part of the digital hologram.

\section{References}

1. G. Antes, Document having an optical-diffraction authenticating element, Swiss patent No. 5820/82, 1993.

2. J.-F. Moser, Document protection by optically variable graphics (Kinegram), Optical Document Security, R. L. van Renesse Ed., Chap. 9, pp. 169-185, Artech House, Boston, 1994.

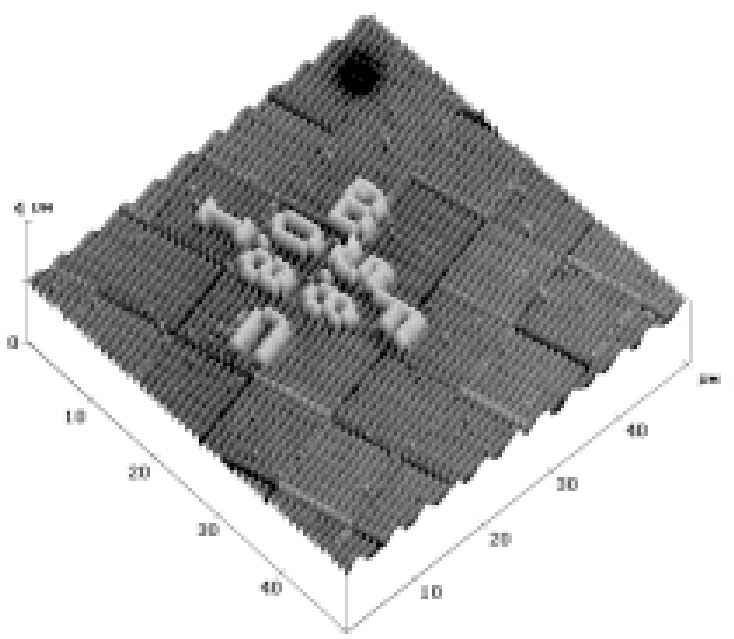

Fig. 8. Microstructure of CO/DSD surface: part of superimposed exposure of optical + digital holograms + submicrotext.

3. R.A. Lee, The Pixelgram - an application of electron bean lithography for the security printing industry // Proc. SPIE 1509, pp. 48-54, 1991.

4. R.A. Lee, Security diffraction grating with special optical effects, Australian patent application No. PL1316, 1993. 